\title{
Discarding duplicate ditags in LongSAGE analysis may introduce significant error
}

\author{
Jeppe Emmersen ${ }^{1}$, Anna M Heidenblut ${ }^{2,3}$, Annabeth Laursen Høgh' ${ }^{1}$ \\ Stephan A Hahn², Karen G Welinder ${ }^{1}$ and Kåre L Nielsen*1
}

Address: ${ }^{1}$ Department of Biotechnology, Chemistry and Environmental Engineering, Aalborg University, Aalborg, Denmark, ${ }^{2}$ Department of Internal Medicine, Knappschaftskrankenhaus, University of Bochum, Bochum, Germany and ${ }^{3}$ Department of Molecular Oncology, Weatherall Institute of Molecular Medicine, Oxford University John Radcliffe Hospital, UK

Email: Jeppe Emmersen - je@bio.aau.dk; Anna M Heidenblut - anna.heidenblut@cancer.org.uk; Annabeth Laursen Høgh - alh@bio.aau.dk; Stephan A Hahn - stephan.hahn@rub.de; Karen G Welinder - kgw@bio.aau.dk; Kåre L Nielsen* - kln@bio.aau.dk

* Corresponding author

Published: 14 March 2007

BMC Bioinformatics 2007, 8:92 doi:10.1/86/|47|-2/05-8-92
Received: 25 September 2006

Accepted: 14 March 2007

This article is available from: http://www.biomedcentral.com/I47I-2/05/8/92

(c) 2007 Emmersen et al; licensee BioMed Central Ltd.

This is an Open Access article distributed under the terms of the Creative Commons Attribution License (http://creativecommons.org/licenses/by/2.0), which permits unrestricted use, distribution, and reproduction in any medium, provided the original work is properly cited.

\begin{abstract}
Background: During gene expression analysis by Serial Analysis of Gene Expression (SAGE), duplicate ditags are routinely removed from the data analysis, because they are suspected to stem from artifacts during SAGE library construction. As a consequence, naturally occurring duplicate ditags are also removed from the analysis leading to an error of measurement.

Results: An algorithm was developed to analyze the differential occurrence of SAGE tags in different ditag combinations. Analysis of a pancreatic acinar cell LongSAGE library showed no sign of a general amplification bias that justified the removal of all duplicate ditags. Extending the analysis to 10 additional LongSAGE libraries showed no justification for removal of all duplicate ditags either. On the contrary, while the error introduced in original SAGE by removal of naturally occurring duplicate ditags is insignificant, it leads to an error of up to 3 fold in LongSAGE. However, the algorithm developed for the analysis of duplicate ditags was able to identify individual artifact ditags that originated from rare nucleotide variations of tags and vector contamination.

Conclusion: The removal of all duplicate ditags was unfounded for the datasets analyzed and led to large errors. This may also be the case for other LongSAGE datasets already present in databases. Analysis of the ditag population, however, can identify artifact tags that should be removed from analysis or have their tag count adjusted.
\end{abstract}

\section{Background}

Serial Analysis of Gene expression (SAGE) is a global and digital gene expression profiling method $[1,2]$. It relies on three fundamental principles: (i) a short nucleotide tag cut from a cDNA copy of an mRNA is sufficient to uniquely identify the transcript, (ii) two tags can be ligated together to form ditags and unambiguously amplified by PCR, and (iii) multiple tags can be concatenated for efficient detection by DNA sequencing. The overall reliability of SAGE has been compared to other gene expression profiling methods such as Northern Blots [3], real-time or kinetic PCR $[4,5]$, and CDNA and oligo nucleotide micro array hybridizations [6-8]. It was generally found that the reliability and reproducibility of SAGE is high. Typically $70-85 \%$ of gene expression changes observed in SAGE can be confirmed by a different method 
$[4,7]$. However, a potential bias introduced by amplification of ditags was discussed already in the original SAGE publication [1]. It was suspected that duplicate ditags, i.e. identical copies of a ditag (AB), would occur only as an artifact of PCR amplification. Therefore, duplicate ditags have been removed prior to tag counting in most SAGE studies so far, partly because of requests from reviewers before publication.

However, duplicate ditags will be encountered naturally with a certain frequency, depending on abundance of the two transcripts from which the ditag is derived $[8,9]$. For example, in the original SAGE protocol two blunt ended 14 nucleotide tags were ligated to form ditags. Two tags A and B, each occurring at a frequency of 0.02 have a 0.0004 probability of being joined. Present SAGE studies typically include 50,000 tags (25,000 ditags) leading to $10 \mathrm{AB}+\mathrm{BA}$ ditags. However, the total count of a tag of 0.02 frequency in 50,000 is 1000 , and the error of 10 introduced by removing the naturally occurring ditags is insignificant. Furthermore, an algorithm to minimize this problem (SAGEparser) was developed by Snyder and coworkers[10].

However, recent developments in SAGE technology have accentuated the problem of discarding duplicate ditags. First, there has been a drive towards using smaller samples for construction of SAGE libraries, facilitating the analysis of cells with specialized functions such as pancreatic cells obtained from biopsies [4]. Such samples may have extreme gene expression profiles with single transcripts accounting for $5 \%$ of the total population of transcripts. Second, the widespread use of the LongSAGE protocol in which a two base pair overhang is used in the ligation of ditags, instead of blunt ends [2]. Consequently, any LongSAGE tag can only form ditags with tags with a compatible overhang, in principle reducing the number of potential partner tags 16 fold on the average. In this paper we analyze the error introduced by discarding naturally occurring duplicate ditags in LongSAGE and describe a probabilistic algorithm that can distinguish naturally occurring ditags from artifacts.

\section{Results and discussion}

A prediction of the number of duplicate ditags as a function of the abundance of the two monotags in SAGE and LongSAGE is shown in figure 1. It illustrates the error introduced by deleting duplicate ditags as commonly practiced in these analyses. In original SAGE, all bluntended tags can combine with all other tags and, therefore, the number of a particular duplicate ditag $\mathrm{AB}$ can be predicted from equation 1. In LongSAGE, tags have a $2 \mathrm{nt}$ overhang and their ditag partner is constrained accordingly. Therefore, the number of a particular LongSAGE ditag can be estimated from equation 2, assuming an even distribution of possible overhangs.

As can be seen in figure 1, discarding duplicate ditags introduces a serious error for abundant tags in LongSAGE
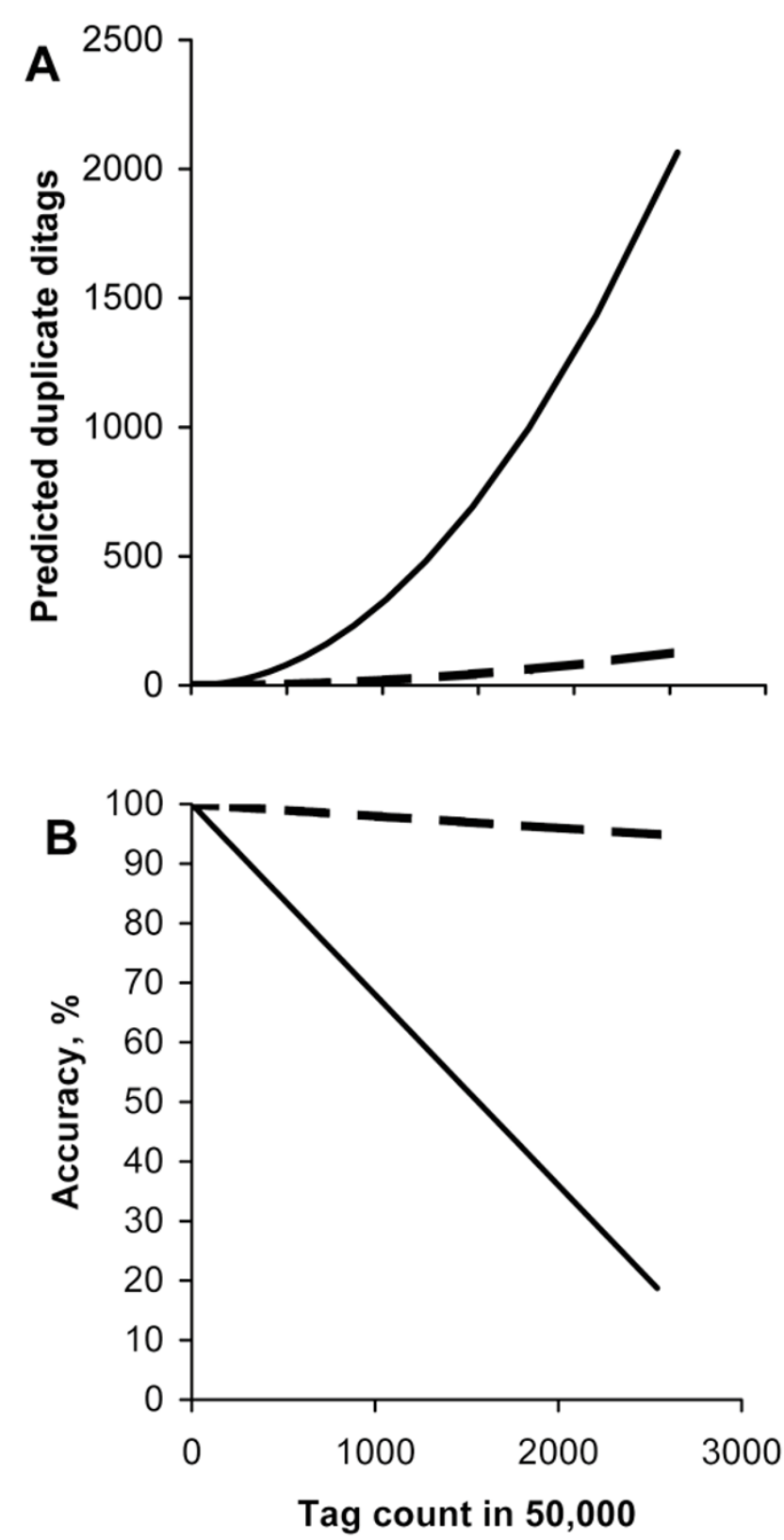

Figure I

Estimating occurrence of duplicate ditags in SAGE based on an even distribution of compatible overlapping tags. (A) The number of expected duplicate ditags (equation I). (B) The accuracy of tag count when duplicate ditags were removed from the analysis. SAGE (hatched line), and LongSAGE (solid line). 
Table I: Abundant LongSAGE tags observed in pancreatic acinar cells.

\begin{tabular}{|c|c|c|c|c|c|}
\hline \multirow[b]{3}{*}{ Tag sequence } & \multicolumn{2}{|c|}{ Duplicate ditags } & \multirow[b]{3}{*}{ Fold change ${ }^{c}$} & \multirow[b]{3}{*}{$\mathrm{THC}^{\mathrm{d}}$} & \multirow[b]{3}{*}{ Gene name } \\
\hline & Includeda & Removed b & & & \\
\hline & Tag count & Tag count & & & \\
\hline CATGTCAGGGTGATTCTGGTG & 3315 & 1086 & 0.33 & 2531342 & Trypsin I \\
\hline CATGGCGTGACCAGCTTTGTT & 2609 & 1161 & 0.44 & 2498325 & Elastase IIIB \\
\hline CATGAATTGAAGAAACTGACC & 2359 & 713 & 0.30 & 2510696 & Unknown \\
\hline CATGGAGCACACCCTGAATCA & 1145 & 657 & 0.57 & 2613307 & Carboxypeptidase AI \\
\hline CATGGAACACAAAAAAAAAAA & 1094 & 535 & 0.49 & & Unknown \\
\hline CATGTGCGAGACCACCCCTAT & 891 & 461 & 0.52 & 2683646 & Carboxypeptidase A2 \\
\hline CATGTCCTCAAAACAAAAAAA & 753 & 377 & 0.50 & & Unknown \\
\hline CATGAGCCTTGGTATCAAGAG & 645 & 353 & 0.55 & 2462969 & Cholesterol esterase \\
\hline CATGTTCATACACCTATCCCC & 531 & 177 & 0.33 & 2398611 & NADH dehydrogenase \\
\hline CATGCTGAATCTAAATTATAA & 526 & 257 & 0.49 & 2590573 & Alpha-amylase 2B \\
\hline CATGTCCTCAAAACAATAAAA & 465 & 252 & 0.54 & & Unknown \\
\hline CATGTCCTCAAAAAAAAAAAA & 431 & 211 & 0.49 & & Unknown \\
\hline
\end{tabular}

aTotal number of tags $=44,276$

bTotal number of tags $=31,868$

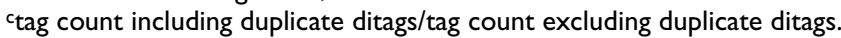

d Tentative Human Contig number from The Institute for Genomic Research

data analysis. For example, two abundant tags, each present 1500 times in a typical 50,000 tag study, would give rise to only 45 duplicate ditags in SAGE, but 720 duplicate ditags in LongSAGE. Counting duplicates only once, as is presently done, would result in an error of 3\% for SAGE and $48 \%$ for LongSAGE. In this example we have removed the duplicates for $\mathrm{AB}$ only, but in reality a particular tag A, will create duplicates with any compatible tag $\mathrm{B}, \mathrm{C}, \mathrm{D}$ etc. at the frequency stipulated in equations 1 or 2 . If each of these duplicates is counted only once, a further reduction of the tag count is introduced and, therefore, the error will increase. Indeed, this simple estimate demonstrates that the problem is expected to be much greater for LongSAGE, than for conventional SAGE.

However, the assumption of equal proportions of compatible overhangs in LongSAGE is unrealistic. The genome sequence is not a random distribution of nucleotides [11] and furthermore, this would limit the maximum tag count of any tag to $1 / 16$ of the total tag count of the library (e.g. 3125 in 50,000), and individual tag counts larger than 3125 have been encountered.

The experimental dataset derived from RNA isolated from pancreatic acinar cells by the aRNA-LongSAGE procedure was therefore analyzed in greater detail [4]. It contains 44,276 tags before removal of duplicate ditags and 31,868 after. The unusual high numbers of duplicate ditags reflects an extraordinary abundance of transcripts encoding the enzymes of the digestive juice (table 1). Table 1 shows the 12 most abundant tags, the enzyme encoded and the tag counts before and after removal of duplicate ditags. Removing duplicate ditags from the dataset reduces the tag count with up to $67 \%$ for the most abun- dant tag. The most abundant tags are the ones most often affected, although medium abundance tags are also significantly affected, if they are compatible with predominant tags (see table 2, and additional file 1). In fact, only for total tag counts (without removal of duplicate ditags) less than 10 is the majority of tags unchanged by removal of duplicate ditags. And 19\% of medium abundance tags (20-49) are changed at least 1.5 fold. Bear in mind, that the error is not affecting all tags to a similar degree, while some tags are unchanged, others may be greatly affected. For an example, the tag count for CATGGGCGACTCTGGCGGCCC is 40 tags before removal and only 14 after, a change of 3 fold [additional file 1]. Anisimov et al. have argued that up to $5 \%$ false ditags, so-called quasi-ditags should be removed from SAGE analyses [12]. In our case, removing quasi-ditags has only marginal effect of the analysis (data not shown), presumably because the error corrected by Anisimov et al. is exclusively affecting rare tags, whereas we are concerning with an error increasingly affecting tags the more abundant they get.

The corrective measures suggested by Welle [9]and Snyder [10] are both iterative approaches developed for SAGE. Welle suggests splitting up the dataset in a number of subdatasets, removing duplicate ditags, and then adding these datasets together allowing duplicates. The number of subdatasets to be created, determining the maximal number of duplicate ditags is a simple guess. In fact, this method is equivalent to setting a maximal allowed ditag count instead of excluding all. In LongSAGE, duplicate ditag counts of several hundreds are frequently observed. Therefore, determining a low meaningful fixed number of duplicate ditags is not feasible. Snyder's algoritm (SAGEparser) includes a proportion of the observed 
duplicate ditags based on the abundance of the two monotags comprising the ditag. This new tag count can then be used to calculate a new proportion of the observed duplicate ditags to be added. Many iterations of this algorithm would approach the inclusion of all duplicate ditags. While this algorithm includes some of the naturally occurring ditags, it only works for SAGE where all tags can form ditags with each other and does not address whether an entire library is biased or not.

In this study, an algorithm implemented in Perl was developed (LongSAGE_bias.pl, see methods for details) which extracts both monotags and ditags from phred or fasta formatted sequence files, defines the two nt overhang of tag pairs in the ditags, and counts and sorts these ditags into compatible overlapping classes. Of the 44,276 tags in total, 34,464 were seen twice or more. Considering these tags only (thus excluding most tags originating from sequencing error) 12,408 (36\%) were present in duplicate ditags. A major complication of the analysis is the presence of most abundant tags in several forms differing in length by one or rarely by two nucleotides. Thus a single tag may be split into two or more compatible overlapping classes. For this analysis, only tags between 40 and $42 \mathrm{nt}$ were considered (including the NlaIII recognition site, CATG of both tags). These accounted for $98.6 \%$ of all ditags (table 3). A 40 nucleotide ditag contains two tags in the short form ( $21 \mathrm{nt}$ each, as $2 \mathrm{nt}$ are shared), a 41 nucleotide ditag one short form and one long form, and a 42 nucleotide ditag contains two tags in the long form (22 nt each, as $2 \mathrm{nt}$ are shared). The relative propensity for a tag to appear in long or short form was calculated for each tag without considering the 41 nucleotide ditag, as for these ditags, we cannot know which of the two tags is present in the long form. Likewise, the compatible overlapping class was determined from the 40 and 42 nucleotide ditags only, because the overlap in these tags can be unambiguously identified as the two central nucleotides of the ditag.

The number of ditags in the 10 possible overlapping classes is tabulated in table 3. The length distribution shows a general overrepresentation of the long form. Apparently, the tag generating restriction enzyme, MmeI, cleaves the DNA strand 21/19 nucleotides downstream of its recognition site twice as often as 20/18 nucleotides. Furthermore, the true distribution among compatible overlapping classes is far from uniform. The overlap CG was only present in 17 ditags, whereas the AA (or TT) was present in 3173 ditags. This is in line with the relative dinucleotide abundance in humans, which also shows a severe under representation of CG, whereas AA (or TT) is more commonly observed [11]. This observation is corroborated with the finding that $\mathrm{CpG}$ islands are predominantly found in the first exon of genes and therefore rarely in the 3' end of transcripts mostly represented in SAGE
[13]. Analyzing the distribution of overlaps generated insilico from the human RefSeq v. 16 also shows a similar tendency, albeit to a lesser extent (table 3).

The uneven distribution of compatible overhangs actually raises the question whether a particular overhang can be present in such a low abundance that it suppresses the measurement of other tags due to an insufficient number of compatible monotags. One solution to this would be to conduct the LongSAGE experiments by blunt-ending by T4-DNA polymerase prior to ligation of ditags, thus shortening the LongSAGE tags by two nucleotides [14]. An advantage of this approach is that the error introduced by removal of duplicate ditags is small and similar to those indicated for SAGE (figure 1). However, in the case presented here, saturation of compatible overlaps is not the reason for the rare occurrence of the CG overlap, as CG is a palindrome and thus may form ditags with itself.

Two predictions are calculated for the occurrence of each ditag (including 41 nucleotide ditags) using equation 3 (see methods and figure 2 for details). The two predictions are often the same, but may differ for ditags containing less abundant tags and will approach uniformity for higher duplicate ditag counts. A plot of the predicted numbers of ditags against the observed number of ditags is shown in figure 3 a [see additional file 2 for details]. The slope of a linear regression analysis of the data is 1.3, in reasonable agreement with the expected 1 . However, the Pearson product moment correlation coefficient between the observed and the predicted is a modest 0.61.

Detection of outliers is performed by calculating standardized residuals according to equation (4) for each ditag. Assuming normal distribution of the standardized residuals, the standard deviation is calculated for all ditags observed more than once. An observation is classified as an outlier if the standardized residual is larger than three standard deviations (99\% confidence). The standardized residuals are plotted in figure $3 \mathrm{~b}$. The low number of standardized residuals that fall outside the confidence interval (outliers) indicates that most duplicate ditags seem to represent true tags.

Inspection of the outliers reveals that most of these ditags contain at least one tag ending in a nucleotide that is inconsistent with the nucleotide sequences of the corresponding Unigene found in the TIGR Human Gene Index (see table 4), and most likely represents nucleotide variations. The overlapping class of this variant tag is inconsistent with the overlapping class of the non-variant tag used for the prediction. Obviously, the algorithm would provide erroneous predictions in these cases, since it is based on the frequency of the non-variant monotag, which is much higher than the frequency of the variant monotag. 
Table 2: The relationship of tag abundance and degree of change introduced by removal of duplicate ditags.

\begin{tabular}{|c|c|c|c|c|c|}
\hline \multirow[t]{2}{*}{ Tag count } & \multirow[t]{2}{*}{ \# of unique tags } & \multicolumn{4}{|c|}{ Observed change upon removal of duplicate ditags } \\
\hline & & $>2$ folda & $1.5-2$ fold ${ }^{a}$ & $\mathrm{I}-\mathrm{I} .5$ fold ${ }^{\mathrm{a}}$ & unchanged $^{a}$ \\
\hline$>200$ & 19 & $7(37)$ & $8(42)$ & $4(2 I)$ & $0(0)$ \\
\hline$>100-199$ & 13 & $3(23)$ & $4(31)$ & $6(46)$ & $0(0)$ \\
\hline$>50-99$ & 42 & $2(5)$ & $13(3 \mid)$ & $27(64)$ & $0(0)$ \\
\hline$>20-49$ & 91 & $6(7)$ & II (I2) & $72(79)$ & $2(2)$ \\
\hline$>10-19$ & 179 & $3(2)$ & $16(9)$ & $104(58)$ & $56(3 I)$ \\
\hline$>5-9$ & 383 & $\mathrm{I}(0.3)$ & $19(5)$ & $139(36)$ & $224(58)$ \\
\hline$>2-5$ & 2157 & $8(0.4)$ & 240 (II) & $120(6)$ & $1789(83)$ \\
\hline
\end{tabular}

a Fold change is calculated by dividing the total tag count with the tag count obtained after removal of duplicate ditags. Percentage of total number of different tags in the indicated intervals is given in parentheses.

A different, rather abundant ditag was observed (86 times) much more often than predicted (8 times). BLAST analysis of this ditag reveals that it consists of two tags derived from the E. coli $\beta$-lactamase gene and thus is most likely the result of vector contamination. Removing these data points from the regression increases the Pearson correlation coefficient to 0.95 . Therefore, an excellent correlation between the number of duplicate ditags observed and predicted is obtained not discarding duplicate ditags.

To investigate whether this is special to this particular dataset, we have performed the analysis on additional datasets, five derived from potato tuber (Høgh, Emmersen and Nielsen, unpublished) and five other libraries derived from pancreatic tissue [4]. The analysis can be carried out on any LongSAGE library, but becomes more precise with more ditags included in the analysis. In our experience, depending on the proportion of duplicate ditags present, a minimum library size of 35,000 tags seems to be the lower limit for a reliable analysis. In the future, exploiting new DNA sequencing technologies, libraries larger than 150,000 tags will probably be common[15]. Varying numbers of duplicate ditags were present in these libraries, and suspicious outlier ditags were identified in all libraries. However, in none of the libraries the duplicate ditags were biased to an extent that justified the bulk removal of all duplicates. Tag extractions including or excluding duplicate ditags of the most abundant tran-

Table 3: Summary of ditag statistics.

\begin{tabular}{lll}
\hline & Pancreatic acinar cells & RefSeq v.16c \\
\hline Ditag length & & \\
\hline 40 & Number & N.A. \\
41 & 3339 & N.A. \\
42 & 11329 & N.A. \\
43 & 7325 & N.A. \\
44 & 240 & N.A. \\
& 61 & Number \\
Overlap class & Number & 5172 \\
\hline ATb & & 1371 \\
CGb & 572 & 3635 \\
GC & 17 & 5076 \\
TA & 344 & 18853 \\
AA or TT & 161 & 8120 \\
AC or GT & 3173 & 11069 \\
AG or CT & 1784 & 10467 \\
CA or TG & 813 & 8561 \\
CC or GG & 437 & 9315 \\
GA or TC & 2868 & \\
\hline
\end{tabular}

aDitags shorter than $\mathbf{4 0}$ nucleotides were not extracted from sequences

bPalindromic sequences. The number of sequences compares to half the number in non-palindromic sequences.

cLongSAGEtags generated in-silico form RefSeq using 17+CATG nt tags only. 


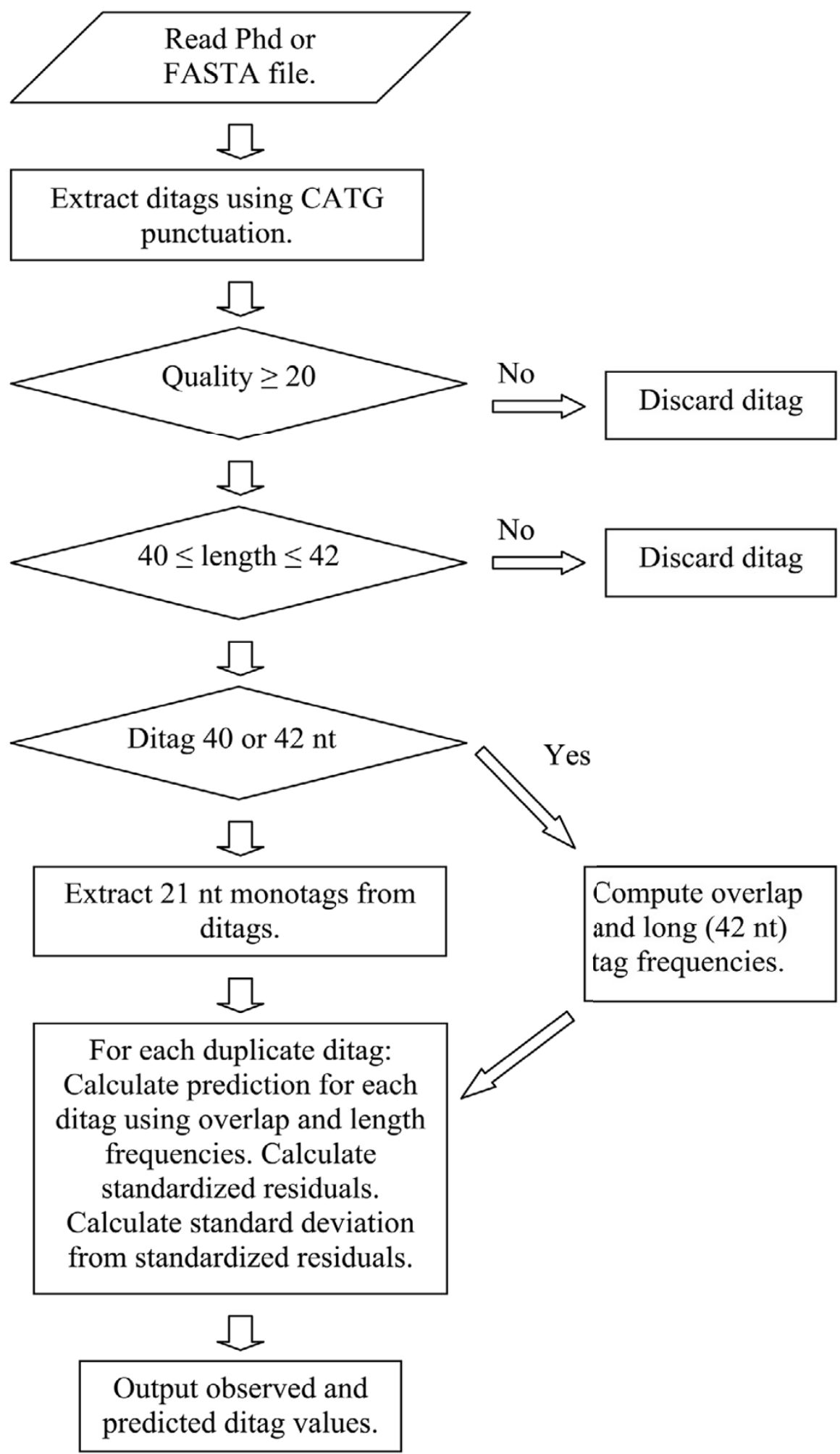

\section{Figure 2}

Overview of the LongSAGE_bias.pI PERL script used for the data analyses. The quality threshold of sequence files can be set at any level desired. A high quality threshold may lead to the under representation of difficult to sequence tags. If set to zero all tags are included and the number of tags observed once or twice increases. 

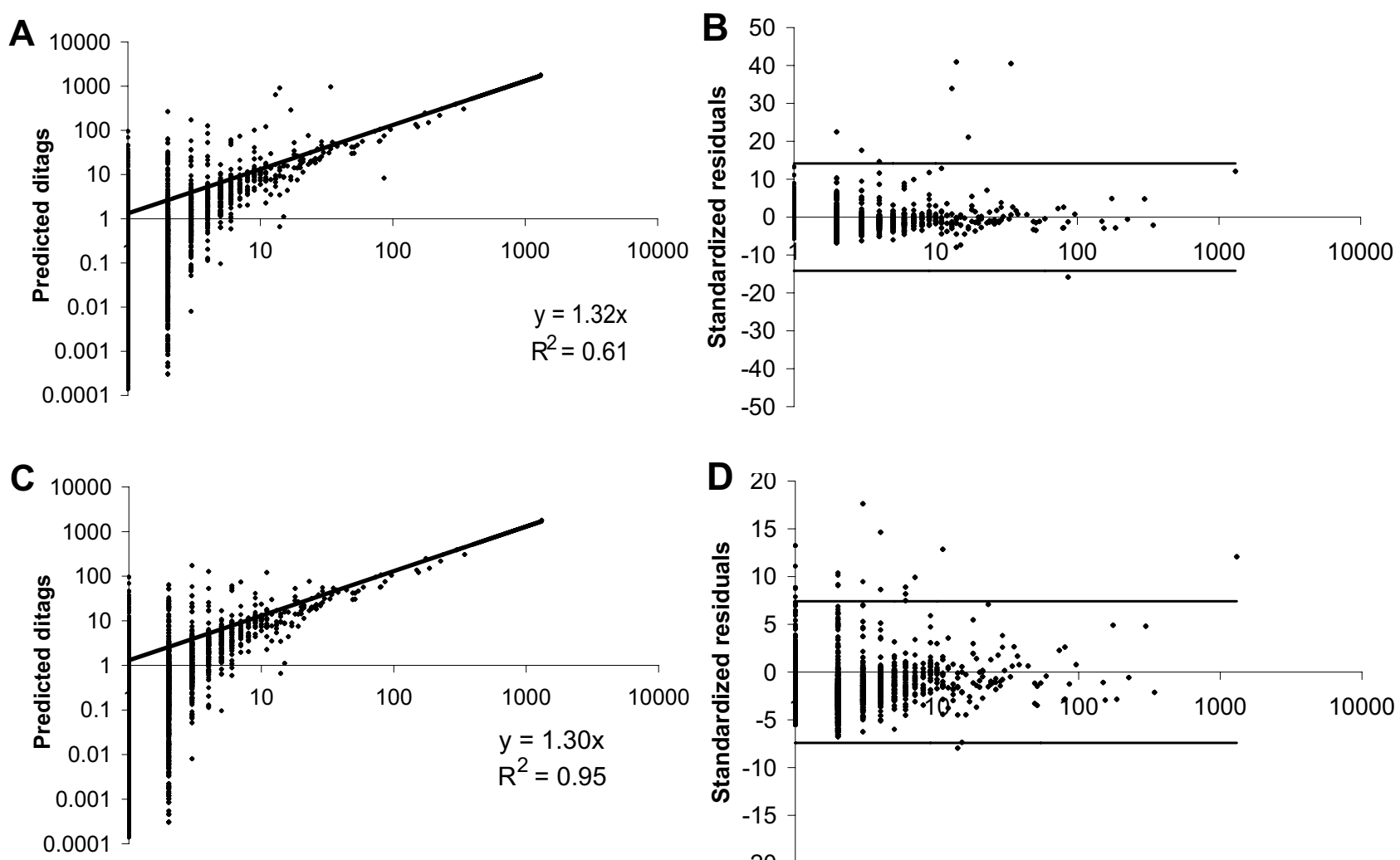

Observed ditags

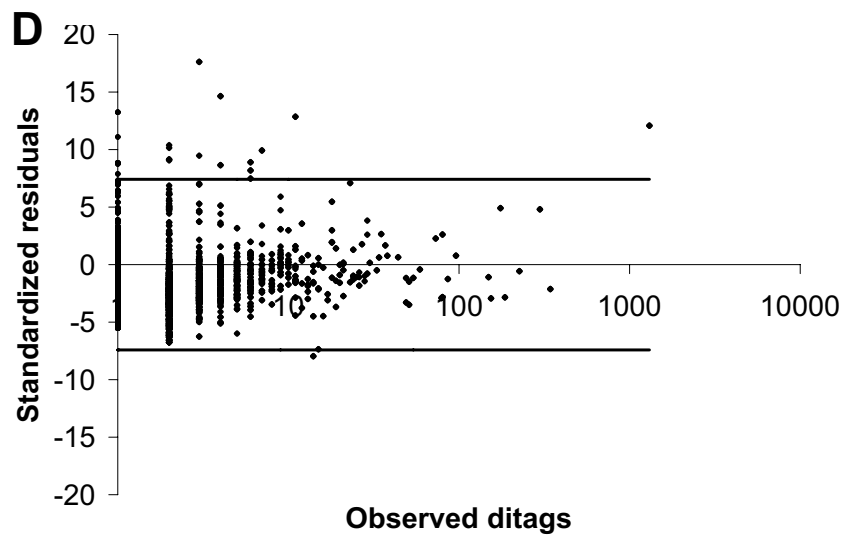

Figure 3

Predicted versus observed duplicate ditags in a LongSAGE study of pancreatic acinar cells [4]. Predicted ditag counts, two for each observed ditag, were calculated according to equation 3 (see methods for details). (A) All observed ditags are included. (C) Outliers, according to table 4 were removed. Standardized residuals were calculated according to equation 4. The confidence interval at three standard deviations is shown as lines. (B) All observed ditags are included. (D) Outliers, according to table 4 were removed. The recalculated confidence interval at three standard deviations is shown as lines.

scripts of all additional 10 libraries analyzed is shown in [additional file 3]. The libraries are affected to a different extent depending on the transcription profile, but all libraries show a change of up to $1.5-2$ folds among the 20 most abundant transcripts, when including or excluding duplicate ditags [see additional file 3]. However, similar to the pancreatic acinar library, the effect was not restricted to high abundance tags, but was observed in medium abundance tags as well (data not shown). The NCBI database, SAGEdB, currently contains 625 SAGE libraries of which 155 are LongSAGE. Unfortunately, ditag sequences are not reported so re-analysis of existing data will have to be carried out by the submitters which hold the original sequence files.

Also, it is important to consider how the removal of duplicate ditags influences the initial identification of a gene as regulated in a comparison of two transcript profiles. To assess whether this changes by exclusion of duplicate ditags, we compared the pancreatic acinar library with one derived from pancreatic ductal cells with and without the inclusion of duplicate ditags. Excluding duplicate ditags, 122 tags was identified as statistically significantly regulated $(\mathrm{P}<0.05$ with Bonferroni correction). Including duplicates yielded 56 new tags, while three fell below the statistical cut-off (additional 43\%) (See table 5). Some of the tags mapped to genes that are known to be highly expressed in acinar but not ductal cells, such as variants of the digestive enzymes chymotrypsin, trypsin and elastase. Therefore, removal of duplicate ditags alters the interpretation of LongSAGE data at least by limiting the power of detecting changes in gene expression; thus effectively excluding what is likely to be valid transcript changes (false negatives) from further analysis and interpretation. 
Table 4: Comparison of outlier ditags with the matched database sequences.

\begin{tabular}{|c|c|c|c|c|}
\hline Obs & Pred & Tag structure & THCa & Gene name \\
\hline \multirow[t]{3}{*}{34} & 970 & CATGGAGCACACCCTGAATCACACCAGAATCACCCTGACATG & & \\
\hline & & CATGGAGCACACCCTGAATCAC & 2401106 & Carboxypeptidase \\
\hline & & CСАCCAGAATCACCCTGACATG & 2434341 & Trypsin I \\
\hline \multirow[t]{3}{*}{17} & 289 & CATGGTGTGTGCTGGAGGGTACACCAGAATCACCCTGACATG & & \\
\hline & & CATGGTGTGTGCTGGAGGGTAC & 2431718 & Elastase IIIA \\
\hline & & CCACCAGAATCACCCTGACATG & 2434341 & Trypsin I \\
\hline \multirow[t]{3}{*}{14} & 913 & CATGTCAGGGTGATTCTGGTGAGGAAGCCCACACAGAACATG & & \\
\hline & & CATGTCAGGGTGATTCTGGTGG & 2434341 & Trypsin I \\
\hline & & AAGGAAGCCCACACAGAACATG & 2434342 & Trypsin I \\
\hline \multirow[t]{3}{*}{13} & 641 & CATGACGCTGGACGCTCCAAGCACCAGAATCACCCTGACATG & & \\
\hline & & CATGACGCTGGACGCTCCAAGC & 2407612 & Colipase \\
\hline & & CCACCAGAATCACCCTGACATG & 2434341 & Trypsin I \\
\hline \multirow[t]{3}{*}{9} & 101 & CATGTCAGGGTGATTCTGGTGTGATTGCCGAGCCAGAGCATG & & \\
\hline & & CATGTCAGGGTGATTCTGGTGG & 2434341 & Trypsin I \\
\hline & & GTGATTGCCGAGCCAGAGCATG & 2237360 & Phospholipase $\mathrm{A} 2^{\mathrm{b}}$ \\
\hline \multirow[t]{3}{*}{4} & 127 & CATGTCAGGGTGATTCTGGTGCTGGCGCTTCTGACCATCATG & & \\
\hline & & CATGTCAGGGTGATTCTGGTGG & 2434341 & Trypsin I \\
\hline & & GCTGGCGCTTCTGACCATCATG & 2401106 & Carboxypeptidase ${ }^{\mathrm{c}}$ \\
\hline \multirow[t]{3}{*}{4} & 85 & CATGACGCTGGACGCTCCAAGTGATTCAGGGTGTGCTCCATG & & \\
\hline & & CATGACGCTGGACGCTCCAAGC & 2407612 & Colipase \\
\hline & & GTGATTCAGGGTGTGCTCCATG & 2401106 & Carboxypeptidase \\
\hline \multirow[t]{3}{*}{2} & 267 & CATGGAGCACACCCTGAATCAAACAAAGCTGGTCACGCCATG & & \\
\hline & & CATGGAGCACACCCTGAATCAC & 2401106 & Carboxypeptidase \\
\hline & & AAACAAAGCTGGTCACGCCATG & 2254617 & Elastase IIIB \\
\hline \multirow[t]{3}{*}{86} & 8 & CATGACAGTAAGAGAATTATGCAGTGCTGCCATAACCATG & & \\
\hline & & CATGACAGTAAGAGAATTATGC & & $\beta$-lactamase \\
\hline & & GCAGTGCTGCCATAACCATG & & Inv. $\beta$-lactamase \\
\hline
\end{tabular}

\footnotetext{
aTentative Human Contig number from The Institute for Genomic Research (TIGR).

bThe match to Phospholipase A2 is not perfect (GTGATTGCCGAGCCAGAGCACG)

cThe tag matches an inverted sequence from carboxypeptidase.
}

It has been found, that the cross-platform agreement of transcriptome measurements by SAGE, LongSAGE and DNA oligonucleotide microarrays was only modest, in contrast to a good intra-platform reproducibility [7]. A majority, but not all of the differentially expressed genes identified by either method can be verified by RT-PCR (e.g. [4] and [16]). Therefore, to maximize the accuracy of LongSAGE and hopefully improve the cross-platform consistency, it is important analyze and adjust the tag sequences (including all duplicate ditags) for a number of potential biases prior to mapping to database sequences. First, linker sequences should be removed. Second, the dataset should be tested with the present algorithm. Duplicate ditag counts that falls within the confidence intervals should not be adjusted. But in the case of outliers, these can be manually removed after careful analysis (as in this study), or automatically adjusted to the predicted count by the algorithm to minimize the impact of artifacts. Third, to minimize the number of false positives identified as differentially expressed genes, artifact tags originating from sequence errors should be resolved using SAGEScreen [17].

\section{Conclusion}

The analyses presented here clearly demonstrate that the present procedure of discarding all duplicate ditags can lead to large errors in LongSAGE studies. Instead, the algorithm described here should be used to test LongSAGE datasets and identify potentially biased ditags that should be adjusted or removed. Based on the results obtained it is likely, that most of the transcriptome profiles present in the databases have been artificially biased by the removal of duplicate ditags.

\section{Methods \\ Equations}

Assuming that the observed tag counts (after amplification and sequence extraction) are representative of the actual distribution of tag molecules, the expected occurrence of a duplicate ditag $\mathrm{AB}$ in SAGE can be approximated by

$$
\mathrm{D}_{\mathrm{AB}, \text { pred }}=\mathrm{D}_{\text {total }} * \mathrm{P}_{(\mathrm{AB})}=\mathrm{D}_{\text {total }} * \mathrm{P}_{(\mathrm{A})} * \mathrm{P}_{(\mathrm{B})}=\mathrm{D}_{\text {total }} * \frac{T_{A}}{T_{\text {total }}} * \frac{T_{B}}{T_{\text {total }}}
$$

where $\mathrm{D}$ is the number of ditags, $\mathrm{P}$ the probability, and $\mathrm{T}$ the number of monotags observed. 
Table 5: Additional transcript changes detected between pancreatic acinar and ductal cells by including duplicate ditags.

\begin{tabular}{|c|c|c|c|c|c|}
\hline Tag & Acinar & Ductal & P-value & Transcript ID ${ }^{a}$ & Gene Name \\
\hline CATGGGCGACTCTGGCGGCCC & 40 & 0 & $6.72 \mathrm{E}-13$ & THC2268952 & Chymotrypsinogen B \\
\hline CATGGAGCACACCCTGAATCC & 39 & 0 & $1.4 \mathrm{E}-12$ & unknown & \\
\hline CATGCCTGTAATCCCAGCTAC & 20 & 95 & $1.23 \mathrm{E}-1 \mathrm{I}$ & W85818 & \\
\hline CATGCCTAGCTGGATTGCAGA & 26 & 104 & 5.89E-II & BU542624 & \\
\hline CATGAAAGTCTAGAAATAAAA & 3 & 41 & $3.62 \mathrm{E}-09$ & THC2400275 & full-length cDNA clone CSODC0I7YH08 \\
\hline CATGCACAAACGGTAGTTTTG & 187 & 110 & $3.78 \mathrm{E}-09$ & AV744668 & \\
\hline CATGTGTGCTAAATGTGTTCG & 69 & 22 & $5.56 \mathrm{E}-09$ & BF08987I & \\
\hline CATGTTCTGTGTGGGCTTCCC & 27 & 0 & 8.83E-09 & unknown & \\
\hline CATGTGCATCTGGTGTAGGAA & 33 & 103 & I.49E-08 & BU626I27 & \\
\hline CATGGGGTTGGCTTGAAACCA & 2 & 35 & I.72E-08 & BG75627I & \\
\hline CATGCACCTCCCACCGGCCGT & 26 & 0 & I.82E-08 & THC2457279 & Elastase $2 \mathrm{~B}$ \\
\hline CATGCTAAGACTTCACCAGTC & 58 & 145 & 2.IE-08 & BU67467I & \\
\hline CATGGTAAGTGTACTGGAAAG & 33 & 4 & $3.3 \mathrm{E}-08$ & THC2400569 & Human mitochondrial genes \\
\hline CATGAATCCTTGCСТСССТСA & 25 & 1 & $3.74 \mathrm{E}-08$ & $\mathrm{~B} 1791939$ & \\
\hline CATGGGAACAAACAGATCGAA & 6 & 44 & 6.7E-08 & NP922813 & CD24 protein \\
\hline CATGGTAATTTAAACAATGAA & 0 & 29 & $7.3 \mathrm{IE}-08$ & THC2336784 & Integrin beta-6 precursor \\
\hline CATGTCCCCGTGGCTGTGGGG & I & 29 & $7.31 \mathrm{E}-08$ & AV700058 & \\
\hline CATGTGCCCTCAGGAAAAAAA & 0 & 29 & $7.3 \mathrm{IE}-08$ & THC2244374 & Neutrophil gelatinase-associated lipocalin \\
\hline CATGGAACACAAAAAAAAAGA & 24 & 0 & $7.68 \mathrm{E}-08$ & unknown & \\
\hline CATGTGGCTTCAAGCCACCAG & 28 & 89 & $8.5 \mathrm{E}-08$ & BF987687 & \\
\hline CATGCCAAACGTGTAACAATT & 7 & 46 & $8.61 \mathrm{E}-08$ & CV350470 & \\
\hline CATGACAGTAAGAGAATTATG & 87 & 39 & I.IIE-07 & unknown & \\
\hline CATGCTGTACAGACACCACCA & 0 & 28 & I.33E-07 & BGI5I226 & \\
\hline CATGGTAAATTTAAAAAAAAA & I & 28 & $1.33 \mathrm{E}-07$ & unknown & \\
\hline CATGAGTTGAAGAAACTGACC & 23 & 0 & I.57E-07 & unknown & \\
\hline CATGGTTATGGCAGCACTGCA & 86 & 39 & I.64E-07 & unknown & \\
\hline CATGGGTGGTGTCTGAGAGGC & 0 & 27 & $2.4 \mathrm{IE}-07$ & THC2256I55 & gastrointestinal glutathione peroxidase 2 \\
\hline CATGTTCATTATAATCTCAAA & 8 & 46 & $2.72 \mathrm{E}-07$ & BG025220 & \\
\hline CATGCATCTTCACCAGCAGCT & 4 & 36 & $2.75 \mathrm{E}-07$ & CD240368 & \\
\hline CATGCTGCTTGGTGAACAATC & 4 & 36 & $2.75 \mathrm{E}-07$ & THC2247807 & Neutral and basic amino acid transport protein \\
\hline CATGTATGACTTAATAAATCC & 2 & 30 & 3.0IE-07 & AA5069II & \\
\hline CATGCTTGTGAACTGCACAAC & 0 & 26 & $4.38 \mathrm{E}-07$ & AA343639 & \\
\hline CATGGAAATTTAAAGCAGGTT & 2 & 29 & $5.3 \mid \mathrm{E}-07$ & THC227204I & \\
\hline CATGCCAGAACAGACTGGTGA & 19 & 67 & $5.89 \mathrm{E}-07$ & CD240292 & \\
\hline CATGCCAGGGTGATTCTGGTG & 21 & 0 & $6.58 \mathrm{E}-07$ & THC2434375 & Trypsin II \\
\hline CATGGTGTGCGCTGGGGGCGT & 21 & 0 & $6.58 \mathrm{E}-07$ & unknown & \\
\hline CATGGATTGAAGAAACTGACC & 21 & 0 & $6.58 \mathrm{E}-07$ & unknown & \\
\hline CATGTGTCCACCATCTCTCTG & 21 & 0 & $6.58 \mathrm{E}-07$ & THC2434352 & Trypsinogen C \\
\hline CATGGCGTGACCAGCTTTGTG & 21 & 1 & $6.58 \mathrm{E}-07$ & unknown & \\
\hline CATGAGCCACTGCGCCCAGCC & 26 & 3 & $6.96 \mathrm{E}-07$ & $\mathrm{H} 75720$ & \\
\hline CATGCTTCTGATCTCAGCAGT & 0 & 25 & $7.92 \mathrm{E}-07$ & THC23I5603 & Heparan sulfate 3-O-sulfotransferase-I \\
\hline CATGCACAGGCAAAATGTATT & I & 25 & $7.92 \mathrm{E}-07$ & CA3।4838 & \\
\hline CATGTGAAGTTATACTGTGGC & 2 & 28 & $9.33 \mathrm{E}-07$ & AW970III & \\
\hline CATGGGATATGTGGTGTATAT & 7 & 41 & $9.67 \mathrm{E}-07$ & AV65676I & \\
\hline CATGCATATCATTAAACAAAT & 5 & 36 & 0.00000106 & NP924865 & Insulin-like growth factor binding protein 7 \\
\hline CATGTATTTTCCAGCTGCCTC & 20 & 1 & 0.00000134 & AA5I4440 & \\
\hline CATGTCAGGGTGGTTCTGGTG & 20 & 1 & 0.00000134 & unknown & \\
\hline CATGTCAGGGTGATCCTGGTG & 20 & 0 & 0.00000134 & unknown & \\
\hline CATGTCAGGGCGATTCTGGTG & 20 & 0 & 0.00000134 & unknown & \\
\hline CATGAAAAGCAGAAATCGGTT & 0 & 24 & 0.00000143 & THC2244965 & Krueppel-like factor 5 \\
\hline CATGTTTGCACCTTTCTAGTT & 0 & 24 & $0.00000 \mid 43$ & NPII 9453 & Connective tissue growth factor \\
\hline CATGATACTTTAATCAGAAGC & I & 24 & 0.00000143 & NPII94I36 & full-length cDNA clone CSODF028YAI9 \\
\hline CATGGCGAAACCCTGTCTCTA & 3 & 30 & 0.00000155 & W03579 & \\
\hline CATGCTTATGGTTGATCAGTT & 2 & 27 & 0.00000164 & CB243786 & \\
\hline CATGCACCTAATTGGAAGCGC & 56 & 22 & 0.00000216 & CFI29I38 & \\
\hline CATGGGAATGTACGTTATTTC & 13 & 52 & 0.00000218 & $N P 1215187$ & mitochondrial ATP synthase \\
\hline
\end{tabular}

aThe percentage of tags idenfied as differentially expressed that cannot be matched to database sequences are similar including (27\%) or excluding (30\%) ditags as well as in this list (24\%). 
The expected occurrence of a duplicate ditag AB in LongSAGE, assuming even distribution of compatible overlapping classes is then (including duplicate ditags).

$$
\mathrm{D}_{\mathrm{AB} \text {,pred }}=\mathrm{D}_{\text {total }} * \frac{\mathrm{T}_{\mathrm{A}}}{\mathrm{T}_{\text {total }}} * \frac{\mathrm{T}_{\mathrm{B}}}{\mathrm{T}_{\text {total }}} * 16
$$

The expected occurrence of a duplicate ditag $\mathrm{AB}$ in LongSAGE, using dataset specific distributions of compatible overlapping classes can be approximated by

$\mathrm{D}_{\mathrm{AB}}=\mathrm{D}_{\text {total }} * \frac{\mathrm{TA} /}{\mathrm{T}_{\text {total }}} * \frac{\mathrm{TB}}{\mathrm{T}_{\mathrm{PPT}}}$

where $\mathrm{T}_{\mathrm{PPT}}$ is the sum of all possible partner tags.

Standardized residuals was calculated as follows [18]

$$
\begin{aligned}
& \mathrm{Y}=\frac{\left(\mathrm{D}_{\text {pred }}+\frac{1}{2}\right)}{D_{\text {pred }}} \\
& \mathrm{T}(\mathrm{Y})=\frac{1-\mathrm{Y}^{2}+2 * \mathrm{Y} * \ln (\mathrm{Y})}{1-\mathrm{Y}^{2}}, \mathrm{~T}(1)=0 \\
& \text { StdRes }=\frac{\left(\mathrm{D}_{\text {obs }}-\mathrm{D}_{\text {pred }}+\frac{2}{3}\right)}{\sqrt{\frac{1+T(Y)}{D_{\text {pred }}}}}
\end{aligned}
$$

\section{Ditag analysis}

A SAGE experiment is performed by digesting cDNA with the frequent cutting restriction enzyme NlaIII, isolating the most 3' fragment and ligating a linker containing the sequence TCCGAC, which is recognized the restriction enzyme MmeI. Tags are generated by MmeI which cleaves the DNA strand 20/18 nt or 21/19 nt downstream of this sequence. Ligated ditags have the general structure CATGXXXXXXXXXXXXXXXX(X)(Y)YYYYYYYYYYYYYYYY-

CATG, where $X$ denotes tag $A$ and $Y$ denote the reverse complement of tag $\mathrm{B}$. The parentheses indicate that most tags exist in both a short and a long form. Hence, the ditag $\mathrm{AB}$ can have the length 40,41 or 42 nucleotides. Two central base pairs are common to both tag A and tag B and originate from the overlap used during ligation. The Perl script, LongSAGEbias.pl [additional file 4] was developed and used for the data analysis (freely available at [19]). A schematic representation of the script is shown in figure 2. First, the script extracts both ditags and monotags (21 nt), including possible linker derived tags, from DNA sequence files and the corresponding quality values (*.phd) generated by the Phred base caller [20]. Second, the script then calculates the length of each ditag and uses the $40 \mathrm{nt}$ and $42 \mathrm{nt}$ ditags for the calculation of the distribution of compatible overlapping tags and the propensi- ties that each tag is 21 (short form) or 22 (long form) nucleotides. This information is then used to predict the occurrence of any ditag composed of tags observed in a duplicate ditag by equation 3 according to the tag length consistent with the ditag in question. For 40 or 42 nucleotide ditags, a prediction is made for each of the two monotags constituting the ditag.

In the case of 41 nucleotide ditags, the ditag $\mathrm{AB}$ is first analyzed. Since A can exist in a 41 nt ditag both in the long and a short form, two predictions are made and the one closest to the observed is chosen. Then, the ditag BA is considered in an identical manner. The standardized residuals are calculated and the results are written to tabulator separated files easily imported into any spreadsheet for further analysis. Assuming the ditag counts are Poisson distributed, the mean can be estimated as the observed count and the standard deviation as the square root of the observed. The confidence interval of ditag counts can thus be estimated as mean $\pm 2 *$ standard deviation. For small ditag counts this confidence interval extends below zero. Consequently, the standard deviation of the standardized residuals is calculated from ditags observed four or more times only $(4-2 * \sqrt{ } 4=0)$.

The algorithm can be set to include all duplicate ditags, remove all duplicate ditags and adjust the observed ditag counts that fall outside the confidence interval to the prediction value.

In sum, libraries derived from pancreatic acinar cells, ductal cells, and four libraries from different grades of pancreatic intraepithelial neoplasia were analyzed from pancreas. In addition, 5 potato tuber libraries derived from 6 week old minitubers, at harvest, two libraries from 60 days post harvest dormant tubers, and from tuber tissue excised from under an emerging sprout.

\section{Analyzing dinucleotide overlap distribution of tags generated in-silico}

LongSAGE tags of $17 \mathrm{nt}+$ CATG were extracted from the human RefSeq v. 16 fasta file and the dinucleotide overlap distributions determined using the PERL script dinuccount.pl [19].

\section{Comparison of LongSAGE libraries with and without inclusion of duplicate ditags}

LongSAGE tags from libraries generated from all potato and pancreatic tissue were extracted using the Perl script sage-phred.pl. For pancreatic acinar and ductal cells the tags were mapped to the Human Gene Index [21] using sagemap.pl. The two libraries were compared using the Perl script acprob.pl and statistically significant changes using strict Bonferroni correction was recorded including 
or excluding duplicate ditags. All scripts are available at [19].

\section{Authors' contributions}

JE, AMH and KLN have designed the analysis of duplicate ditags. JE have produced scripts and performed the analysis. SAH and AMH have performed the LongSAGE studies on pancreatic tissue. ALH carried out the LongSAGE analyses on the potato. KLN drafted the manuscript, which was extensively discussed and modified by KLN, AMH, JE and KGW. Finally, all authors read and approved the final manuscript.

\section{Additional material}

\section{Additional File 1}

Pancreatic acinar LongSAGE. Additional file 1 contains the tag counts of the pancreatic acinar LongSAGE library described in the manuscript with and without duplicate ditags.

Click here for file

[http://www.biomedcentral.com/content/supplementary/14712105-8-92-S1.txt]

\section{Additional File 2}

Observed and predicted LongSAGE ditags of pancreatic acinar cells. Additional file 2 contains the data that constitutes figure 3 and is the background for table 1 and 2.

Click here for file

[http://www.biomedcentral.com/content/supplementary/1471-

2105-8-92-S2.txt]

\section{Additional File 3}

Most abundant transcripts with and without the removal of duplicate ditags. Additional file 3 contains the top20 transcripts of the ten LongSAGE libraries discussed in the text with and without duplicate ditags.

Click here for file

[http://www.biomedcentral.com/content/supplementary/14712105-8-92-S3.txt]

\section{Additional File 4}

longsagebias.pl. This file contains the PERL script that performs the ditag analysis described.

Click here for file

[http://www.biomedcentral.com/content/supplementary/1471-

2105-8-92-S4.pl]

\section{Acknowledgements}

The authors would like to thank Poul Svante Eriksen, Department of Mathematical Sciences, Aalborg University for valuable help with the statistics and Sabine Burkert, Birgit Strzeletzki and Susanne Braun for excellent technical assistance preparing the pancreatic acinar library. This work was supported by the Danish Veterinarian and Agricultural Research Council (2302-0034) and the Danish Technical Research Council (26-00-0I4I). A.M.H. and S.A.H. were supported by grants from the Deutsche Krebshilfe (702988-Schm3), the Bundesministerium für Bildung und Forschung (03 I I 878) and by EU-Grant BMH4-QLGI-CT-2002-01 196.

\section{References}

I. Velculescu VE, Zhang L, Vogelstein B, Kinzler KW: Serial analysis of gene expression. Science 1995, 270:484-487.

2. Saha S, Sparks AB, Rago C, Akmaev V, Wang CJ, Vogelstein B, Kinzler $\mathrm{KW}$, Velculescu VE: Using the transcriptome to annotate the genome. Nat Biotechnol 2002, 20:508-5I2.

3. Velculescu VE, Zhang L, Zhou W, Vogelstein J, Basrai MA, Bassett DE Jr., Hieter P, Vogelstein B, Kinzler KW: Characterization of the yeast transcriptome. Cell 1997, 88:243-25I.

4. Heidenblut AM, Luttges J, Buchholz M, Heinitz C, Emmersen J, Nielsen KL, Schreiter P, Souquet M, Nowacki S, Herbrand U, Kloppel G, Schmiegel W, Gress T, Hahn SA: aRNA-longSAGE: a new approach to generate SAGE libraries from microdissected cells. Nucleic Acids Res 2004, 32:e I 31.

5. Kang JJ, Watson RM, Fisher ME, Higuchi R, Gelfand DH, Holland MJ: Transcript quantitation in total yeast cellular RNA using kinetic PCR. Nucleic Acids Res 2000, 28:e2.

6. Anisimov SV, Tarasov KV, Stern MD, Lakatta EG, Boheler KR: A quantitative and validated SAGE transcriptome reference for adult mouse heart. Genomics 2002, 80:2 13-222.

7. van RF, Ruijter JM, Schaaf GJ, Asgharnegad L, Zwijnenburg DA, Kool $M$, Baas $F$ : Evaluation of the similarity of gene expression data estimated with SAGE and Affymetrix GeneChips. BMC Genomics 2005, 6:91.

8. Dinel S, Bolduc C, Belleau P, Boivin A, Yoshioka M, Calvo E, Piedboeuf B, Snyder EE, Labrie F, St-Amand J: Reproducibility, bioinformatic analysis and power of the SAGE method to evaluate changes in transcriptome. Nucleic Acids Res 2005, 33:e26.

9. Welle S, Bhatt K, Thornton CA: Inventory of high-abundance mRNAs in skeletal muscle of normal men. Genome Res 1999, 9:506-5I3.

10. SAGEParser Home Page 2007 [http://obesitygene.pbrc.edu/ حeesnyder/sageparser.htm].

II. Gentles AJ, Karlin S: Genome-scale compositional comparisons in eukaryotes. Genome Res 200 I, I I:540-546.

12. Anisimov SV, Sharov AA: Incidence of "quasi-ditags" in catalogs generated by Serial Analysis of Gene Expression (SAGE). Bmc Bioinformatics 2004, 5:.

13. Venter JC, Adams MD, Myers EW, Li PW, Mural RJ, Sutton GG, Smith HO, Yandell M, Evans CA, Holt RA, Gocayne JD, Amanatides P, Ballew RM, Huson DH, Wortman JR, Zhang Q, Kodira CD, Zheng XH, Chen L, Skupski M, Subramanian G, Thomas PD, Zhang J, Gabor Miklos GL, Nelson C, Broder S, Clark AG, Nadeau J, McKusick VA, Zinder N, Levine AJ, Roberts RJ, Simon M, Slayman C, Hunkapiller M, Bolanos R, Delcher A, Dew I, Fasulo D, Flanigan M, Florea L, Halpern A, Hannenhalli S, Kravitz S, Levy S, Mobarry C, Reinert K, Remington K, bu-Threideh J, Beasley E, Biddick K, Bonazzi V, Brandon R, Cargill M, Chandramouliswaran I, Charlab R, Chaturvedi K, Deng Z, Di F V, Dunn P, Eilbeck K, Evangelista C, Gabrielian AE, Gan W, Ge W, Gong F, Gu Z, Guan P, Heiman TJ, Higgins ME, Ji RR, Ke Z, Ketchum KA, Lai Z, Lei Y, Li Z, Li J, Liang Y, Lin X, Lu F, Merkulov GV, Milshina N, Moore HM, Naik AK, Narayan VA, Neelam B, Nusskern D, Rusch DB, Salzberg S, Shao W, Shue B, Sun J, Wang Z, Wang A, Wang X, Wang J, Wei M, Wides R, Xiao C, Yan C, Yao A, Ye J, Zhan M, Zhang W, Zhang H, Zhao Q, Zheng L, Zhong F, Zhong W, Zhu S, Zhao S, Gilbert D, Baumhueter S, Spier G, Carter C, Cravchik A, Woodage $\mathrm{T}$, Ali F, An H, Awe A, Baldwin D, Baden H, Barnstead M, Barrow I, Beeson K, Busam D, Carver A, Center A, Cheng ML, Curry L, Danaher S, Davenport L, Desilets R, Dietz S, Dodson K, Doup L, Ferriera S, Garg N, Gluecksmann A, Hart B, Haynes J, Haynes C, Heiner C, Hladun S, Hostin D, Houck J, Howland T, lbegwam C, Johnson J, Kalush F, Kline L, Koduru S, Love A, Mann F, May D, McCawley S, McIntosh T, McMullen I, Moy M, Moy L, Murphy B, Nelson K, Pfannkoch C, Pratts E, Puri V, Qureshi H, Reardon M, Rodriguez R, Rogers YH, Romblad D, Ruhfel B, Scott R, Sitter C, Smallwood M, Stewart E, Strong R, Suh E, Thomas R, Tint NN, Tse S, Vech C, Wang G, Wetter J, Williams S, Williams M, Windsor S, Winn-Deen E, Wolfe K, Zaveri J, Zaveri K, Abril JF, Guigo R, Campbell MJ, Sjolander KV, Karlak B, Kejariwal A, Mi H, Lazareva B, Hatton T, Narechania A, Diemer K, Muruganujan A, Guo N, Sato S, Bafna V, Istrail S, Lippert R, Schwartz R, Walenz B, Yooseph S, Allen D, Basu A, Baxendale J, Blick L, Caminha M, Carnes-Stine J, Caulk P, Chiang YH, Coyne M, Dahlke C, Mays A, Dombroski M, Donnelly M, Ely D, Esparham S, Fosler C, Gire H, Glanowski S, Glasser K, Glodek A, Gorokhov M, Graham K, Gropman B, Harris M, Heil J, Henderson S, Hoover J, Jennings D, Jordan C, Jordan J, Kasha J, Kagan L, Kraft C, Levitsky A, Lewis M, Liu X, Lopez 
J, Ma D, Majoros W, McDaniel J, Murphy S, Newman M, Nguyen T, Nguyen N, Nodell M: The sequence of the human genome. Science 200I, 29 I: I304-I35I.

14. Nielsen KL, Grønkjær K, Welinder KG, Emmersen J: Global transcript profiling of potato tuber using LongSAGE. Plant Biotechnology Journal 2005, 3:175-185.

15. Nielsen KL, Hogh AL, Emmersen J: DeepSAGE--digital transcriptomics with high sensitivity, simple experimental protocol and multiplexing of samples. Nucleic Acids Res 2006, 34: I 33.

16. Dallas PB, Gottardo NG, Firth MJ, Beesley AH, Hoffmann K, Terry PA, Freitas JR, Boag JM, Cummings AJ, Kees UR: Gene expression levels assessed by oligonucleotide microarray analysis and quantitative real-time RT-PCR -- how well do they correlate? BMC Genomics 2005, 6:59.

17. Akmaev VR, Wang CJ: Correction of sequence-based artifacts in serial analysis of gene expression. Bioinformatics 2004, 20:1254-1263.

18. Kotz S, B.Read C, N.Balakrishnan, Vidakovic B: Encyclopedia of Statistical Sciences Hoboken, New Jersey, USA, John Wiley \& Sons; 2006:23-23.

19. Aalborg University SAGE software 2007 [http:// www.bio.aau.dk/en/biotechnology/software applications].

20. Ewing B, Hillier L, WendI MC, Green P: Base-calling of automated sequencer traces using phred. $I$. Accuracy assessment. Genome Res 1998, 8:175-185.

21. The Institute for Genome Research 2007 [http://www.tigr.org].

\section{Publish with Bio Med Central and every scientist can read your work free of charge}

"BioMed Central will be the most significant development for disseminating the results of biomedical research in our lifetime. "

Sir Paul Nurse, Cancer Research UK

Your research papers will be:

- available free of charge to the entire biomedical community

- peer reviewed and published immediately upon acceptance

- cited in PubMed and archived on PubMed Central

- yours - you keep the copyright

Submit your manuscript here:

http://www.biomedcentral.com/info/publishing_adv.asp 